\title{
Correspondence
}

\section{Minimum Error Probability MIMO-Aided Relaying: Multihop, Parallel, and Cognitive Designs}

\author{
Amit Kumar Dutta, Member, IEEE, K.V.S. Hari, Fellow, IEEE, \\ Chandra R. Murthy, Senior Member, IEEE, \\ Neelesh B. Mehta, Senior Member, IEEE, \\ and Lajos Hanzo, Fellow, IEEE
}

\begin{abstract}
A design methodology based on the minimum error probability (MEP) framework is proposed for a nonregenerative multiple-input multiple-output relay-aided system. We consider the associated cognitive, the parallel, and the multihop source-relay-destination link design based on this MEP framework, including the transmit precoder, the amplify-andforward relay matrix, and the receiver equalizer matrix of our system. It has been shown in the literature that MEP-based communication systems are capable of improving the error probability of other linear counterparts. Our simulation results demonstrate that the proposed scheme indeed achieves a significant bit-error-ratio reduction over the existing linear schemes.
\end{abstract}

Index Terms-Cognitive, linear minimum mean square error (LMMSE), maximization of the capacity (MC), minimum error probability (MEP), multiple-input multiple-output (MIMO), relay.

\section{INTRODUCTION}

Multiple-input multiple-output (MIMO) relaying is becoming an eminent and integral part of advanced wireless communication systems [1], owing to its capability of enhancing the received signal. The joint design of the transmitter of the relay and of the destination receiver along with the MIMO benefits has attracted tremendous research attention [1], [2]. New MIMO-aided relay configurations, namely multihop relays, parallel relays, and a relay-aided cognitive, have been considered by numerous researchers for tackling a range of challenges, including the coverage range extension [3], [4] and the careful choice of the best links from the entire set of legitimate links [5].

Numerous design criteria, such as the mean square error (MSE), the maximization of the capacity (MC), and various others, have been used for MIMO-aided relaying in the literature. For example, multihop relaying, which is capable of substantially extending the cellular coverage, has been designed relying on the MSE criterion [3], [4]. On the other hand, the so-called parallel relay configuration [5], which allows the best relay link to be selected from a set of parallel relay links, used the MSE criterion for designing the relaying weights. Cognitive communications, where the bandwidth is judiciously shared between the primary and secondary users, has also been extended to the family of MIMO relay-aided systems [6], [7] using the MC criterion. However, a fundamental limitation of these criteria is that they are unable to achieve the minimum error probability (MEP), i.e., the lowest bit error ratio (BER) in a linear detection framework [8]. Hence, the MEP-based transceiver

Manuscript received August 17, 2015; revised August 8, 2016; accepted September 26, 2016. Date of publication; date of current version. The review of this paper was coordinated by Dr. Y. Ma.

A. K. Dutta, K. V. S. Hari, C. R. Murthy, and N. B. Mehta are with the Department of Electrical Communication Engineering, Indian Institute of Science, Bangalore 560012, India (e-mail: amitkdutta6@gmail.com; hari@ece. iisc.ernet.in; cmurthy@ece.iisc.ernet.in; neeleshbmehta@gmail.com).

L. Hanzo is with the School of Electronics and Computer Science, University of Southampton, Southampton SO17 1BJ, U.K. (e-mail: 1h@ecs.soton.ac.uk).

Digital Object Identifier 10.1109/TVT.2016.2614901

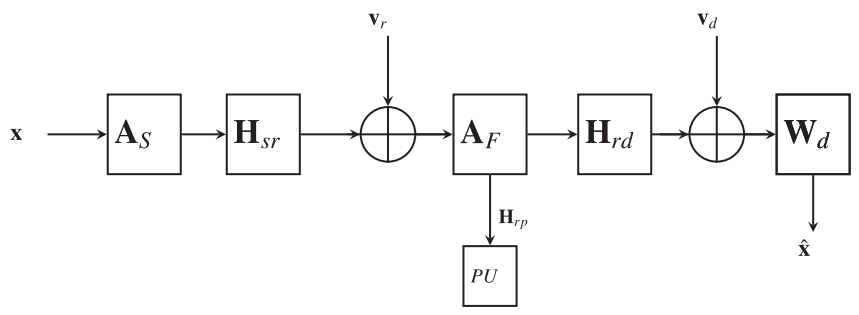

Fig. 1. Cognitive MIMO-relay system.

design criterion, also known as the minimum BER (MBER) method, is a more pertinent design criterion as far as the BER performance is concerned. Although the benefits of the MEP-based MIMO-relaying system have already been demonstrated in [9] in terms of an SNR gain of up to $3-4 \mathrm{~dB}$, in this treatise, our holistic $\mathrm{CF}$ is conceived in the above mentioned scenarios equipped with MIMO configurations for the first time.

Against this background, the contributions of this treatise are as follows. We propose to invoke the MEP optimization criterion as our objective function for jointly optimizing the transmit precoder (TPC) at the source, the amplify-and-forward (AF) MIMO weights at the relays, and the equalizer weights at the destination of three different relaying topologies-namely the multihop, the parallel, and the cognitive relaying regimes. We develop the MEP-based cost function (CF) for these three network topologies based on the classic quadrature phase-shift keying (QPSK) signal constellation. We opted for the projected steepest descent (PSD) [10] optimization tool for finding the minimum of the CF. Our numerical simulations demonstrate that this criterion leads to significantly lower BER than its counterparts.

Our system model is presented in Section II, followed by the formulation of the MEP CF in Section III and by our numerical results in Section IV, before concluding in Section V.

\section{SYSTEM MODEL}

In the following, we present the system model of the abovementioned three topologies, namely the cognitive, parallel, and multihop relay configurations separately.

\section{A. Cognitive MIMO-Relay Model}

For the cognitive MIMO relay, we consider a single-hop relaying system consisting of a source node ( $\mathrm{SN})$, a relay node (RN), and a destination node (DN) having $N_{s}, N_{r}$, and $N_{d}$ antennas, respectively, as shown in Fig. 1. Let us assume that the primary user (PU), sharing the same bandwidth and having $N_{p}$ receiver antenna, suffers from interference from RN [6]. Let us denote that $N_{x}$ is the length of the input vector $\mathbf{x} \in \mathbb{C}^{N_{x} \times 1}$ before the TPC operation at the SN, where $\mathbf{A}_{s} \in \mathbb{C}^{N_{s} \times N_{x}}$ is the TPC matrix. We denote $\mathbf{H}_{s r} \in \mathbb{C}^{N_{r} \times N_{s}}, \mathbf{H}_{r d} \in$ $\mathbb{C}^{N_{d} \times N_{r}}$, and $\mathbf{H}_{r p} \in \mathbb{C}^{N_{d} \times N_{r}}$ as the SN-RN, RN-DN, and SN-PU channel gain matrices, respectively. Let us denote the independent and identically distributed (i.i.d) additive white Gaussian noise vectors at the $\mathrm{RN}$ and $\mathrm{DN}$ as $\mathbf{v}_{r} \in \mathbb{C}^{N_{r} \times 1}$ and $\mathbf{v}_{d} \in \mathbb{C}^{N_{d} \times 1}$, with the variance of $\sigma_{r}^{2}$ and $\sigma_{d}^{2}$ for each component, respectively. Thus, the vector received at the $\mathrm{RN}$ is given by

$$
\mathbf{r}_{r}=\mathbf{H}_{s r} \mathbf{A}_{S} \mathbf{x}+\mathbf{v}_{r} .
$$




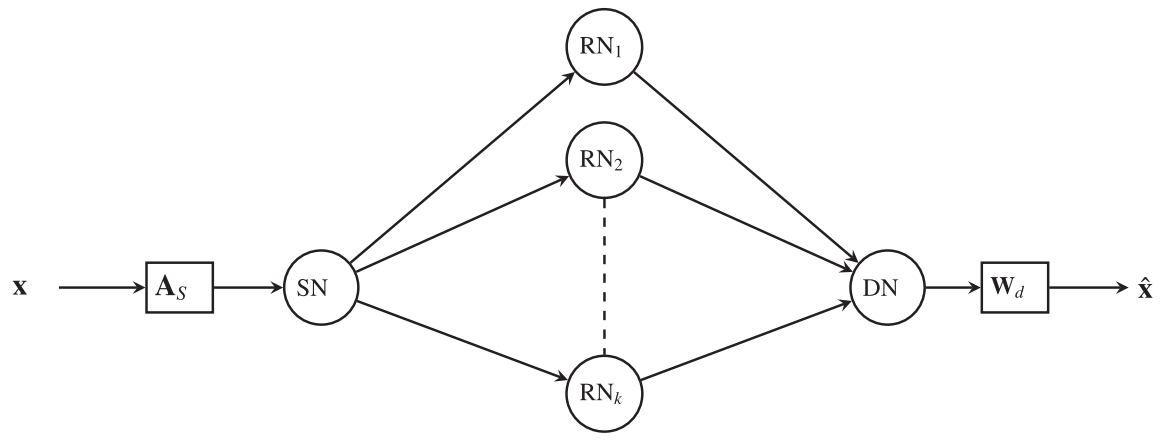

Fig. 2. Parallel MIMO-relay system.

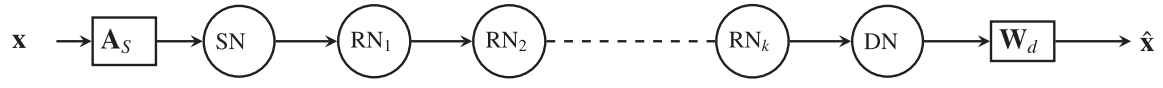

Fig. 3. Multihop MIMO-relay system.

Let us denote the AF matrix by $\mathbf{A}_{F} \in \mathbb{C}^{N_{r} \times N_{r}}$. The power constraint at the $\mathrm{RN}$ is calculated as

$$
\operatorname{Tr}\left[\mathbf{A}_{F}\left(\sigma_{x}^{2} \mathbf{H}_{s r} \mathbf{A}_{S} \mathbf{A}_{S}^{H} \mathbf{H}_{s r}^{H}+\sigma_{r}^{2} \mathbf{I}_{N_{r}}\right) \mathbf{A}_{F}^{H}\right] \leq P_{r}
$$

where $P_{r}$ is the RN's transmit power and $\mathbb{E}\left\{\mathbf{x x}^{H}\right\}=\sigma_{x}^{2} \mathbf{I}_{N_{x}}$. We also calculate the average interference $\left(I_{p}\right)$ at the $\mathrm{PU}$ as

$$
\operatorname{Tr}\left[\mathbf{H}_{r p} \mathbf{A}_{f} \mathbf{A}_{f}^{H} \mathbf{H}_{r p}^{H}+\rho_{1} \mathbf{H}_{r p} \mathbf{A}_{f} \mathbf{H}_{s r} \mathbf{A}_{s} \mathbf{A}_{s}^{H} \mathbf{H}_{s r}^{H} \mathbf{A}_{f}^{H} \mathbf{H}_{r p}^{H}\right] \leq I_{p} / \sigma_{r}^{2}
$$

where $\rho_{1}=I_{p} / \sigma_{r}^{2}$. Similarly, we obtain the received signal at the DN as

$$
\begin{aligned}
\mathbf{r}_{d} & =\mathbf{H}_{r d} \mathbf{A}_{F} \mathbf{H}_{s r} \mathbf{A}_{S} \mathbf{x}+\mathbf{H}_{r d} \mathbf{A}_{F} \mathbf{v}_{r}+\mathbf{v}_{d} \\
& \triangleq \mathbf{H} \mathbf{x}+\mathbf{v}
\end{aligned}
$$

where $\mathbf{H} \triangleq \mathbf{H}_{r d} \mathbf{A}_{F} \mathbf{H}_{s r} \mathbf{A}_{S}$ and $\mathbf{v} \triangleq \mathbf{H}_{r d} \mathbf{A}_{F} \mathbf{v}_{r}+\mathbf{v}_{d}$, while $\mathbf{v}_{d}$ is the noise at DN, which has a covariance matrix of $\sigma_{d}^{2} \mathbf{I}_{N_{d}}$. The effective noise $\mathbf{v}$ has a covariance matrix of $\mathbf{C}_{v}=\sigma_{d}^{2} \mathbf{I}_{N_{d}}+\mathbf{H}_{r d} \mathbf{A}_{F} \mathbf{A}_{F}^{H} \mathbf{H}_{r d}^{H}$. An equalizer matrix $\mathbf{W}_{d} \in \mathbb{C}^{N_{d} \times N_{x}}$ used at the DN would estimate the vector $\mathbf{x}$ by $\hat{\mathbf{x}}=\mathbf{W}_{d}^{H} \mathbf{r}_{d}$.

\section{B. Parallel MIMO-Relay Model}

For the parallel MIMO relay, our final design goal is to select the best relay link from the set of parallel relay links between the $\mathrm{SN}$ and the DN, as shown in Fig. 2. We assume that there are $K$ parallel relays between the source and destination. Let us denote the channel matrices between the $\mathrm{SN}$ and the $k$ th relay as well as the $k$ th relay and the $\mathrm{DN}$, respectively, by $\mathbf{H}_{s r}^{k}$ and $\mathbf{H}_{r d}^{k}$. Furthermore, we denote the AF matrix at the $k$ th $\mathrm{RN}$ by $\mathbf{A}_{F, k}$. The data received at the $k$ th relay after multiplication by the AF relaying matrix are given by

$$
\mathbf{r}_{r, k}=\mathbf{A}_{F} \mathbf{H}_{s r, k} \mathbf{A}_{S} \mathbf{x}+\mathbf{A}_{F, k} \mathbf{v}_{r, k}
$$

with the power constraint formulated as

$$
\operatorname{Tr}\left[\mathbf{A}_{F, k}\left(\sigma_{x}^{2} \mathbf{H}_{s r, k} \mathbf{A}_{S} \mathbf{A}_{S}^{H}\left(\mathbf{H}_{s r, k}\right)^{H}+\sigma_{r}^{2} \mathbf{I}_{N_{r}}\right)\right] \leq P_{r} .
$$

We assume that each link has a maximum power budget of $P_{r}$. The data received at the $\mathrm{DN}$ from the $k$ th relay link are given by

$$
\mathbf{r}_{d, k}=\mathbf{H}_{r d, k} \mathbf{A}_{F, k} \mathbf{H}_{s r, k} \mathbf{A}_{S} \mathbf{x}+\mathbf{H}_{r d, k} \mathbf{A}_{F, k} \mathbf{v}_{r, k}+\mathbf{v}_{d} .
$$

\section{Multihop MIMO-Relay Model}

For the multihop MIMO-relay scenario, we assume that there are $K \quad 112$ recursive single relays, as shown in Fig. 3. For simplicity, we assume 113 having a single source and a DN. The matrices $\mathbf{H}_{r, k} \in \mathbf{C}^{N_{r} \times N_{r}}$ and 114 $\mathbf{A}_{F, k} \in \mathbf{C}^{N_{r} \times N_{r}}$ represent the $(k-1)$ th to $k$ th relay link and the AF 115 relaying matrix of the $k$ th $\mathrm{RN}$, respectively. We impose the power 116 constraint of $P_{r, k}$ at the $k$ th RN. Hence, the signal received at the $k$ th 117 $\mathrm{RN}$ after multiplication by the AF relaying matrix becomes [3], [4]

$$
\mathbf{r}_{f, k}=\prod_{i=1}^{k}\left(\mathbf{H}_{r, i} \mathbf{A}_{F, i}\right) \mathbf{A}_{S} \mathbf{x}+\sum_{j=2}^{k}\left[\prod_{i=1}^{k}\left(\mathbf{H}_{r, i} \mathbf{A}_{F, i}\right) \mathbf{v}_{r, j-1}\right]+\mathbf{v}_{r, k} .
$$

Similarly, the signal received at the $\mathrm{DN}$ is given by

$$
\begin{aligned}
& \mathbf{r}_{d}=\mathbf{H}_{r d} \mathbf{A}_{F, K} \prod_{k=1}^{K-1}\left(\mathbf{H}_{r, i} \mathbf{A}_{F, k}\right) \mathbf{A}_{S} \mathbf{x}+ \\
& \mathbf{H}_{r d, K-1} \mathbf{A}_{F, K-1} \times\left[\sum_{j=2}^{K-1}\left[\prod_{i=1}^{K-1}\left(\mathbf{H}_{r, i} \mathbf{A}_{F, i}\right) \mathbf{v}_{r, j-1}\right]+\mathbf{v}_{r, K-1}\right]+\mathbf{v}_{d} \\
& \triangleq \mathbf{H} \mathbf{x}+\mathbf{v}
\end{aligned}
$$

where $\mathbf{H}$ and $\mathbf{v}$ are defined as follows:

$$
\begin{aligned}
\mathbf{H} \triangleq & \mathbf{H}_{r d} \mathbf{A}_{F, K} \prod_{k=1}^{K-1}\left(\mathbf{H}_{r, i} \mathbf{A}_{F, i}\right) \mathbf{A}_{S} \\
\mathbf{v} \triangleq & \mathbf{H}_{r d, K-1} \mathbf{A}_{F, K-1} \\
& \times\left[\sum_{j=2}^{K-1}\left[\prod_{i=1}^{K-1}\left(\mathbf{H}_{r, i} \mathbf{A}_{F, i}\right) \mathbf{v}_{r, j-1}\right]+\mathbf{v}_{r, K-1}\right]+\mathbf{v}_{d} .
\end{aligned}
$$

The overall covariance matrix is then defined as

$$
\mathbf{C}_{v}=\sum_{k=2}^{K} \sigma_{k}^{2}\left(\prod_{i=k}^{K} \mathbf{H}_{r, i} \mathbf{A}_{F, i}\right)\left(\prod_{i=k}^{K} \mathbf{H}_{r, i} \mathbf{A}_{F, i}\right)^{H}+\sigma_{d}^{2} \mathbf{I}_{N_{d}}
$$

We assume that the channel state information (CSI) is required at 122 various nodes as depicted in Table I. We assume that DN and the PU 123 send the CSI to the RN through feedback channel. 
TABLE I

REQUiREMENT OF CSI AT VARIOUS NODES FOR THE MEP -CRITERION-BASED RELAY DESIGN

\begin{tabular}{lccc}
\hline \hline Link & SN & RN & DN \\
\hline SN-RN-DN & $\mathbf{H}_{s r}, \mathbf{H}_{r d}, \mathbf{H}_{r p}$ & $\mathbf{H}_{r d}$ \\
\hline \hline
\end{tabular}

\section{III. $\mathrm{MEP} \mathrm{CF}$}

In the current context, the MEP CF directly minimizes the BER of the system at the DN. We formulate the MEP CF for the QPSK constellation for the sake of conceptual simplicity. Let us denote the symbol error ratio (SER) by $P_{e, i}$, when detecting $x_{i}$ (the $i$ th component of $\mathbf{x}$ ) at the DN. With a slight "abuse" of notation, we consider the SER here instead of BER, since the BER and SER are approximately related to each other as $\mathrm{SER} \approx \log _{2}(M) \times \mathrm{BER}$ in conjunction with gray coding. If every $x_{i}$ is detected independently, the average probability of a symbol error associated with detecting the complete vector $\mathrm{x}$ is given by

$$
P_{e}=\frac{1}{N_{x}} \sum_{i=1}^{N_{x}} P_{e, i}
$$

Let us denote $\mathbf{w}_{i}$ as the $i$ th column of the DN's equalizer matrix $\mathbf{W}_{d}$. Assume that $L=2^{N_{x}}$ represents the total number of unique realizations of $\mathbf{x}$, while $\mathbf{x}_{j}$ is the $j$ th such realization of $\mathbf{x}$. For the Gaussian $Q(x)$ function, we use an approximation, which works well for a good range of $x$. This is given as [11]

$$
Q(x)=K_{c} \exp \left(-\frac{m_{c} x^{2}}{2}\right)
$$

where $m_{c}$ is chosen from $1 \leq x \leq 2$ and $K_{c}$ is function of $m_{c}$ as defined in [11]. If $\hat{x}_{i}$ is the estimate of $x_{i}$ for the QPSK constellation, we arrive at the expression of $P_{e, i}$ in (15) [9]

$$
\begin{aligned}
P_{e, i}= & \frac{1}{2} \mathbb{E}_{\mathbf{x}}\left[Q\left(\frac{\Re\left[\left(\mathbf{w}_{i}\right)^{H} \mathbf{H x}\right] \Re\left\{x_{i}\right\}}{\sqrt{\frac{1}{2}\left(\mathbf{w}_{i}\right)^{H} \mathbf{C}_{v} \mathbf{w}_{i}}}\right)\right] \\
& +\frac{1}{2} \mathbb{E}_{\mathbf{x}}\left[Q\left(\frac{\Im\left[\left(\mathbf{w}_{i}\right)^{H} \mathbf{H x}\right] \Im\left\{x_{i}\right\}}{\sqrt{\frac{1}{2}\left(\mathbf{w}_{i}\right)^{H} \mathbf{C}_{v} \mathbf{w}_{i}}}\right)\right] \\
= & \frac{1}{L} \sum_{j=1}^{L} Q\left(\frac{\Re\left[\left(\mathbf{w}_{i}\right)^{H} \mathbf{H} \mathbf{x}_{j}\right] \Re\left\{x_{i}\right\}}{\sqrt{\frac{1}{2}\left(\mathbf{w}_{i}\right)^{H} \mathbf{C}_{v} \mathbf{w}_{i}}}\right) \\
& +\frac{1}{L} \sum_{j=1}^{L} Q\left(\frac{\Im\left[\left(\mathbf{w}_{i}\right)^{H} \mathbf{H} \mathbf{x}_{j}\right] \Im\left\{x_{i}\right\}}{\sqrt{\frac{1}{2}\left(\mathbf{w}_{i}\right)^{H} \mathbf{C}_{v} \mathbf{w}_{i}}}\right) \\
\approx & \frac{K_{c}}{L} \sum_{j=1}^{L} \exp \left(-\frac{m_{c} a_{1}^{2}}{2}\right)+\frac{1}{L} \sum_{j=1}^{L} \exp \left(-\frac{m_{c} a_{2}^{2}}{2}\right),
\end{aligned}
$$

where $a_{1}=\frac{\Re\left[\left(\mathbf{w}_{i}\right)^{H} \mathbf{H} \mathbf{x}_{j}\right] \Re\left\{x_{i}\right\}}{\sqrt{\frac{1}{2}\left(\mathbf{w}_{i}\right)^{H} \mathbf{C}_{v} \mathbf{w}_{i}}}$ and $a_{2}=\frac{\Im\left[\left(\mathbf{w}_{i}\right)^{H} \mathbf{H x}_{j}\right] \Im\left\{x_{i}\right\}}{\sqrt{\frac{1}{2}\left(\mathbf{w}_{i}\right)^{H} \mathbf{C}_{v} \mathbf{w}_{i}}}$.

\section{A. Optimization Problem}

We now have to obtain the optimal TPC weights as well as the AF and equalizer matrices by optimizing the CF. Hence, for the cognitive
TABLE II

COMPUTATION COMPLEXITY COMPARISON BETWEEN THE PROPOSED MEP Methods (Multihop And Cognitive) With Existing LMMSE Method

\begin{tabular}{ll}
\hline \hline Type of Relay & \multicolumn{1}{c}{ Approximate complexity number } \\
\hline Cognitive & $N_{\text {itn }}\left(3 \min \left(N_{d}, N_{r}, N_{s}\right)\right.$ \\
& $+2 N_{d} N_{s}+\left(22 N_{r}-2\right) N_{r} N_{d}+4 N_{d}^{2}$ \\
& $+N_{s}\left(8 N_{d}^{2}+17 N_{d}\right)+4 N_{d} N_{s} N_{s}$ \\
& $\left.+6 N_{s} 4^{N} N_{Q}+18 N_{r}+N_{s}+12+N_{d}\right)$ \\
Multihop & $N_{\text {itn }}\left(K\left(14 N_{r}^{2}+N_{s} N_{d}\right)\right.$ \\
& $+4 N_{d} N_{s} N_{x}+4 N_{s} N_{d}^{2}+2 N_{s} N_{d}$ \\
& $+\left(32 K N_{d}^{3}+60 K N_{d}^{2}-14 N_{d}\right) / 3$ \\
& $+\left(8 N_{s}-2\right) N_{d} N_{s}+\left(8 N_{d}-2\right) N_{s} N_{d}$ \\
\hline \hline
\end{tabular}

The result is QPSK dataset with $K$ relays.

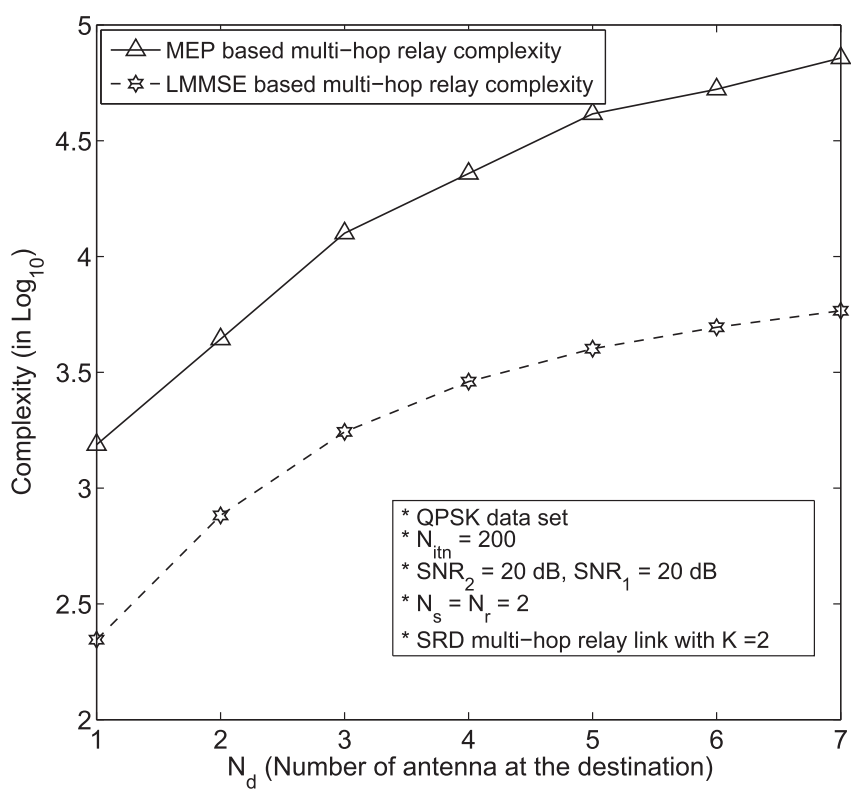

Fig. 4. Typical complexity comparison between the LMMSE and MEP methods for multihop relay design, varying the $N_{d}$ only.

TABLE III

SIMULATION PARAMETERS

\begin{tabular}{lc}
\hline \hline Parameter Name & Values \\
\hline$N_{x}, N_{s}, N_{r}, N_{d}, N_{p}$ & 2 \\
$P_{t}$ (Each relay link) & $0 \mathrm{dBm}, 10 \mathrm{dBm}$ \\
$P_{r}$ (E)stellation & $5 \mathrm{dBm}$ \\
SNR $_{1}$ (Each Relay link) & QPSK \\
$K$ & 4(Parallel), 2(Multihop) \\
\hline
\end{tabular}

case, the optimization problem can be stated as

$$
\begin{aligned}
& \mathbf{A}_{S}^{\text {mep }}, \mathbf{A}_{F}^{\text {mep }}, \mathbf{W}_{d}^{\text {mep }}=\underset{\mathbf{A}_{S}, \mathbf{A}_{F}, \mathbf{W}_{d}}{\arg } \min P_{e}\left(\mathbf{A}_{S}, \mathbf{A}_{F}, \mathbf{W}_{d}\right) \\
& \text { s.t (1) } \operatorname{Tr}\left[\mathbf{A}_{F}\left(\sigma_{x}^{2} \mathbf{C}_{r}^{-1} \mathbf{H}_{s r} \mathbf{A}_{S} \mathbf{A}_{S}^{H} \mathbf{H}_{s r}^{H}\left(\mathbf{C}_{r}^{H}\right)^{-1}+\mathbf{I}_{N_{r}}\right) \mathbf{A}_{F}^{H}\right] \leq P_{r} \\
& \text { (2) } \sigma_{x}^{2} \operatorname{Tr}\left\{\mathbf{A}_{S}^{H} \mathbf{A}_{S}\right\} \leq P_{t} \\
& \text { (3) } \operatorname{Tr}\left[\mathbf{H}_{r p} \mathbf{A}_{f} \mathbf{A}_{f}^{H} \mathbf{H}_{r p}^{H}+\rho_{1} \mathbf{H}_{r p} \mathbf{A}_{f} \mathbf{H}_{s r} \mathbf{A}_{s} \mathbf{A}_{s}^{H} \mathbf{H}_{s r}^{H} \mathbf{A}_{f}^{H} \mathbf{H}_{r p}^{H}\right] \\
& \quad \leq I_{p} / \sigma_{r}^{2} .
\end{aligned}
$$




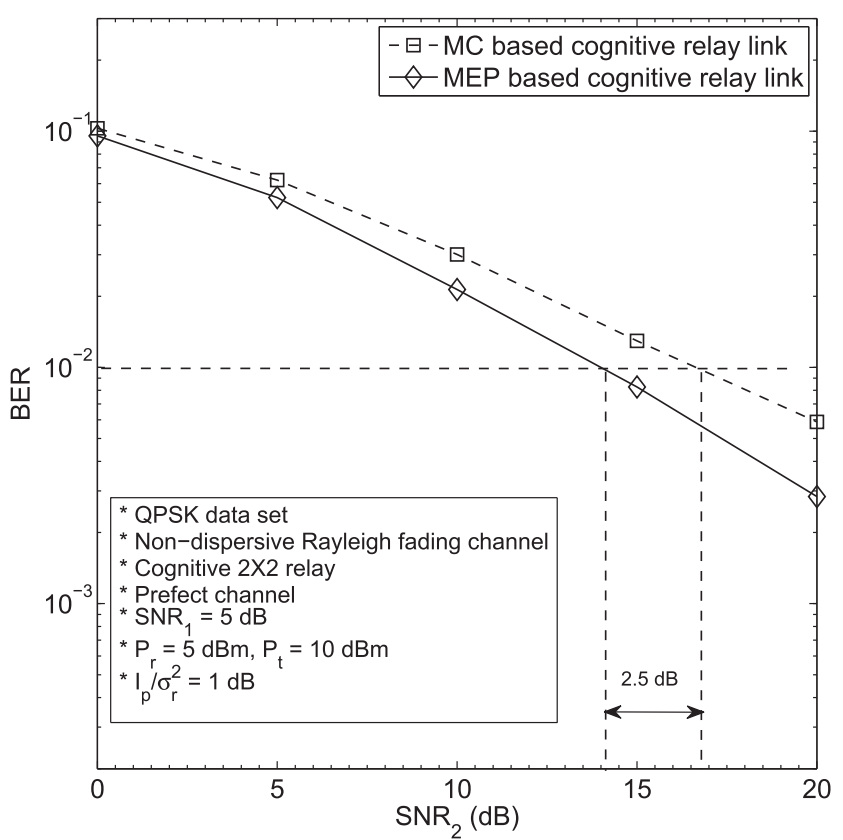

(a)

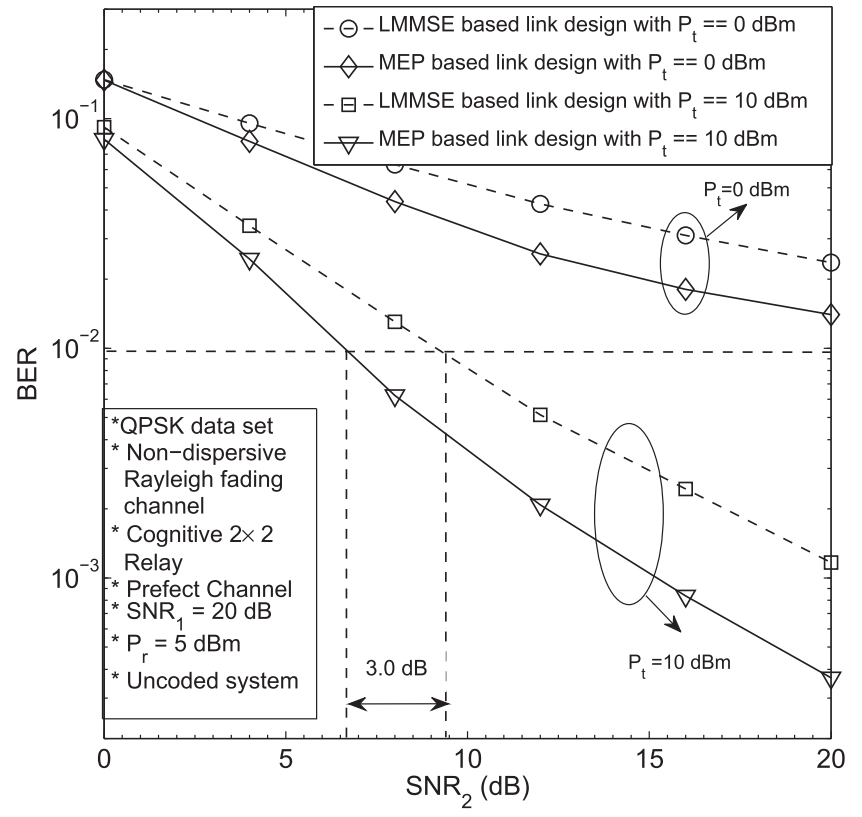

(b)

Fig. 5. BER versus $\mathrm{SNR}_{2}$ performance of the SRD link design for a cognitive MIMO relay based on the MEP method along with the MC method [6] over a flat Rayleigh fading channel without the channel estimation. $N_{s}, N_{r}, N_{d}, N_{p}=2, P_{r}$ is constrained to $5 \mathrm{dBm}$ as shown in Table III. (a) BER performance with $P_{t}=10 \mathrm{dBm}$ and $\mathrm{SNR}_{1}$ is kept at $5 \mathrm{~dB}$. (b) BER performance with $P_{t}=0,10 \mathrm{dBm}$.

For the parallel relaying case, this is a two-step process. In the first step, we optimize each parallel link independently as per equation similar to (16), and then, during the second step, we choose the specific link having the lowest value of the $\mathrm{CF}$, i.e., the lowest $P_{e}$. For the multihop relaying case, the optimization problem is stated as follows:

$$
\begin{aligned}
\mathbf{A}_{S}^{\text {mep }}, \mathbf{A}_{F, k}^{\text {mep }}, \mathbf{W}_{d}^{\text {mep }}=\underset{\mathbf{A}_{S}, \mathbf{A}_{F, k}, \mathbf{W}_{d}}{\arg } \min P_{e}\left(\mathbf{A}_{S}, \mathbf{A}_{F, k} \mathbf{W}_{d}\right) \\
\text { s.t (1) } \operatorname{Tr}\left\{\mathbf{A}_{F}\left(\sigma_{x}^{2} \mathbf{H}_{s r} \mathbf{H}_{s r}^{H}+\sigma_{r}^{2} \mathbf{I}_{N_{r}}\right) \mathbf{A}_{F}^{H}\right\} \leq P_{r, k} \\
\text { (2) } \sigma_{x}^{2} \operatorname{Tr}\left\{\mathbf{A}_{S}^{H} \mathbf{A}_{S}\right\} \leq P_{t},(\text { for } k=1,2, \ldots, K .)
\end{aligned}
$$

In the literature, both gradient and bioinspired solutions [12] have been invoked for optimization problems specific to MEP framework [9]. Here, we have opted for the PSD [10] for solving our constrained optimization problem, because it was found beneficial in [9]. The initial condition for all of them is chosen to be the linear minimum mean square error (LMMSE) solution except for the cognitive case, where an MC-based initial solution is chosen. This is because unless the matrices involved are strongly rank deficient and hence noninvertible, it is reasonable to assume that the MEP solution will be in this neighborhood [9]. For the case of multihop relaying, even the simplest LMMSE solution has no closed-form expression. Hence, in that case, we opted for using a random initial condition for the LMMSE case and invoked the LMMSE solution for the MEP based one.

\section{B. Computational Complexity}

Let us now approximate the computational complexity of the relay link designs using the MEP CF. We characterize it in terms of the number of operations, which can be additions, subtractions, and multiplications. The results have been extrapolated from [9]. For the case of parallel relaying, the results remain similar to [9], except we need to incur an additional cost of $O \log K$ for searching the best link. Hence,

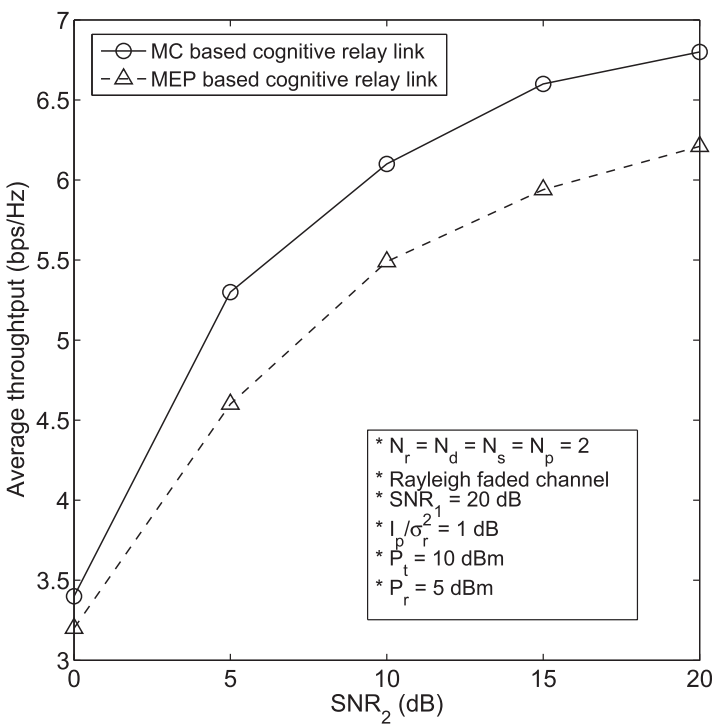

Fig. 6. Capacity comparison for MEP- and MC-based cognitive system with $\mathrm{SNR}_{1}=20 \mathrm{~dB}$.

we present the complexity results only for the cognitive and for the multihop relaying.

Let us assume that $N_{Q}$ represents the approximate number of operations required for computing the $Q(\cdot)$ function, which can be accurately approximated as Taylor series. The computational complexity of the LMMSE solution conceived for the multihop scenario has not been analyzed in the literature. We approximate it as $N_{\text {itn }}\left(K\left(8 N_{s}-2\right) N_{s}^{2}+29 N_{s}+3+K\left(8 N_{r}-2\right) N_{r}^{2}+\right.$ $2 N_{r}+\left(8 N_{s}-2\right) N_{r} N_{s}+\left(32 N_{s}^{3}+60 N_{s}^{2}-14 N_{s}\right) / 3+\left(8 N_{s}-2\right)$ $N_{d} N_{s}+\left(8 N_{d}-2\right) N_{s} N_{d}+2 N_{s} N_{d}+4 N_{d}^{2}+\left(32 N_{d}^{3}+60 N_{d}^{2}-\right.$ 


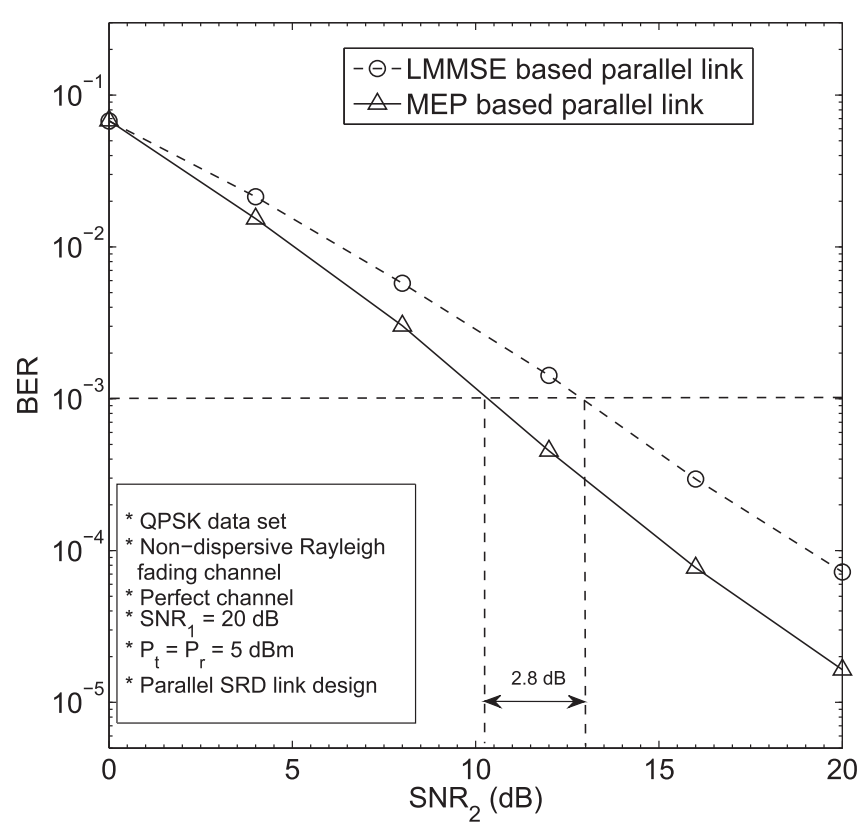

(a)

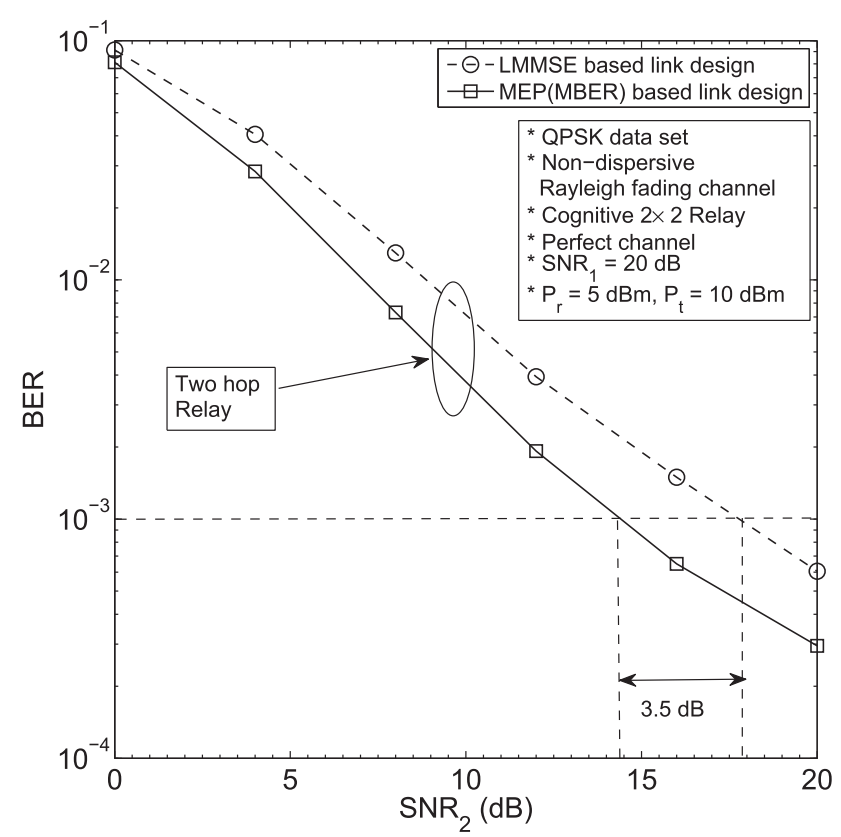

(b)

Fig. 7. BER versus $\mathrm{SNR}_{2}$ performance of the SRD link design for a parallel and a multihop relay systems. The parameters are defined in Table III. (a) BER versus $\mathrm{SNR}_{2}$ performance of the SRD link design for a 4-parallel MIMO relay based on the MEP method along with the LMMSE method over a flat Rayleigh fading channel. $N_{s}, N_{r}, N_{d}=2, P_{r}$ at each RN is constrained to $5 \mathrm{dBm}$ and $\mathrm{SNR}_{1}$ is $20 \mathrm{~dB}$ as shown in Table III. (b) BER versus SNR 2 performance of the SRD link design for a multihop MIMO relay link based on the MEP method along with the LMMSE method over a flat Rayleigh fading channel. $N_{s}, N_{r}, N_{d}=2$, $P_{r}$ at each $\mathrm{RN}$ is constrained to $5 \mathrm{dBm}$ and $\mathrm{SNR}_{1}$ is $20 \mathrm{~dB}$ as shown in Table III.

$\left.14 N_{d}\right) / 3+3 \min \left(N_{d}, N_{r}, N_{s}\right) 2 N_{d} N_{s}+K\left(8 N_{r}-2\right) N_{r} N_{d}+$ $N_{d}$ ), where $N_{\text {itn }}$ is the average number of iterations used by our optimization method. Note that even the LMMSE solution has no closed-form expression for the multihop scenario. Finally, the complexity is presented in Table II.

A typical comparison curve is presented in Fig. 4 for the multihop relay design varying $N_{d}$.

\section{NUMERICAL RESULTS}

Let us now study the BER performance of the proposed method against LMMSE/MC methods for all the above-mentioned MIMOrelay configurations. We consider a nondispersive Rayleigh fading i.i.d channel with unit variance for each complex element of the channel matrix of the various links. We have used perfect channel for our simulation. The RN's SNR is defined as $\mathrm{SNR}_{1}=10 \log _{10}\left(\frac{\sigma_{x}^{2}}{\sigma_{1}^{2}}\right) \mathrm{dB}$, where $\sigma_{x}^{2}$ is the power of each $x_{i}$, which is set to $\frac{P_{t}}{N_{x}}$. The DN's SNR is defined as $\mathrm{SNR}_{2}=10 \log _{10}\left(\frac{P_{r}}{N_{r} \sigma_{2}^{2}}\right) \mathrm{dB}$. The $\mathrm{SNR}_{1}$ is kept at $20,5 \mathrm{~dB}$. $I_{p} / \sigma_{r}^{2}=1 \mathrm{~dB}$. Our simulation results are averaged over 1000 channel realizations per SNR value. We summarize the simulation parameters in Table III.

In this work, we have designed only the SN-RN-DN link of the various configurations.

1) Cognitive relay: This characterizes our cognitive relay link design based on the BER performance of the proposed MEP method against that of the MC benchmarker [6]. It can be observed in Fig. 5(a) $\left(\mathrm{SNR}_{1}=5 \mathrm{~dB}\right)$ that the MEP method achieves a BER of $10^{-2}$ at the SNR of $\approx 14.2 \mathrm{~dB}$, whereas its MC counterpart achieves the same BER at the SNR of $\approx 16.7 \mathrm{~dB}$. Hence, the MEPbased relay design attains an overall SNR gain of about $2.5 \mathrm{~dB}$ at the BER of $10^{-2}$. This gain is further increased for higher SNRs. As expected, the BER performance is poorer for $P_{t}=0 \mathrm{dBm}$, as observed in Fig. 5(b). Fig. 6 shows a capacity comparison. We observe that the capacity of the MEP method is poorer as expected.

2) Parallel relay: This solution relies on finding the best link from the set of parallel relay links using $K=4$. For each link, we have kept the total relay power at $5 \mathrm{dBm}$. It can be observed in Fig. 7(a) that the MEP method attains the BER of $10^{-3}$ at the SNR of about $10.2 \mathrm{~dB}$, whereas its LMMSE counterpart achieves the same BER at the $\mathrm{SNR}$ of $\approx 13 \mathrm{~dB}$. Hence, the MEP-based relay design attains an overall SNR gain of about $\approx 2.8 \mathrm{~dB}$ at the BER of $10^{-3}$.

3) Multihop relay: Let us now embark on characterizing a multihop MIMO relay link. We opted for $N_{r}=2$ for all the intermediate RNs. We have chosen $K=2$, i.e., two serial relay links. For each link, we have kept the total relay power at $5 \mathrm{dBm}$. It can be observed in Fig. 7(b) that the MEP method attains the BER of $10^{-3}$ at the SNR of about $14.5 \mathrm{~dB}$, whereas its LMMSE counterpart achieves the same BER at the SNR of $\approx 18 \mathrm{~dB}$. Hence, the MEP-based relay design attains an overall SNR gain of almost $3.5 \mathrm{~dB}$ at the BER of $10^{-3}$.

\section{CONCLUSION}

In this treatise, we have extended the MEP-based framework to the design of various types of relaying configurations. We have considered cognitive, parallel, and multihop relaying. CFs have been developed and optimization frameworks have been conceived. Numerical simulations have shown considerable BER performance improvements in all these cases. Future research will have to be focused on reducing the computational complexity. 


\section{REFERENCES}

[1] Y. Rong, X. Tang, and Y. Hua, "A unified framework for optimizing linear nonregenerative multicarrier MIMO relay communication systems," IEEE Trans. Signal Process., vol. 57, no. 12, pp. 4837-4851, Dec. 2009.

[2] Y. Rong, "Optimal linear non-regenerative multi-hop MIMO relays with MMSE-DFE receiver at the destination," IEEE Trans. Wireless Commun., vol. 9, no. 7, pp. 2268-2279, Jul. 2010.

[3] Y. Hu, K. H. Li, and K. C. Teh, "An efficient successive relaying protocol for multiple-relay cooperative networks," IEEE Trans. Wireless Commun., vol. 11, no. 5, pp. 1892-1899, May 2012.

[4] M. R. A. Khandaker and Y. Rong, "Transceiver optimization for multi-hop MIMO relay multicasting from multiple sources," IEEE Trans. Wireless Commun., vol. 13, no. 9, pp. 5162-5172, Sep. 2014.

[5] E. Basar, U. Aygolu, E. Panayyrcy, and H. V. Poor, "A reliable successive relaying protocol," IEEE Trans. Commun., vol. 62, no. 5, pp. 1431-1443, May 2014

[6] K. R. Budhathoki, M. Maleki, and H. R. Bahrami, "Iterative source and relay precoder design for non-regenerative MIMO cognitive relay systems," IEEE Trans. Commun., vol. 63, no. 10, pp. 3497-3510, Oct. 2015.
[7] K. R. Budhathoki, M. Maleki, and H. R. Bahrami, "Precoder design for 258 non-regenerative MIMO relay cognitive radio systems," in Proc. IEEE 259 78th Veh. Technol. Conf., Sep. 2013, pp. 1-6.

[8] S. Chen, A. Livingstone, and L. Hanzo, "Minimum bit-error rate design 261 for space-time equalization-based multiuser detection," IEEE Trans. Com- 262 mun., vol. 54, no. 5, pp. 824-832, May 2006.

[9] A. Dutta, K. Hari, and L. Hanzo, "Linear transceiver design for an amplify- 264 and-forward relay based on the MBER criterion," IEEE Trans. Commun., 265 vol. 62, no. 11, pp. 3765-3777, Nov. 2014.

[10] D. H. Luenberger, Linear and Nonlinear Programming. Englewood Cliffs, 267 NJ, USA: Prentice-Hall, 1984.

[11] F. D. Cote, I. N. Psaromiligkos, and W. J. Gross, "A Chernoff-type lower bound for the Gaussian Q-function," arxiv.org/abs/1202.6483v2, 270 Mar. 2012.

[12] D. E. Goldberg, Genetic Algorithms in Search, Optimization, and Machine 272 Learning. Boston, MA, USA: Addison-Wesley Longman, 2009. 